\title{
Cardiomyocyte overexpression of iNOS in mice results in peroxynitrite generation, heart block, and sudden death
}

\author{
Imran N. Mungrue, ${ }^{1,2,3}$ Robert Gros, ${ }^{1,2}$ Xiaomang You, ${ }^{1,2}$ Asif Pirani, ${ }^{2}$ Azar Azad, ${ }^{4}$ \\ Tamas Csont, ${ }^{5}$ Richard Schulz, ${ }^{5}$ Jagdish Butany, ${ }^{1}$ Duncan J. Stewart, $, 1,3,4$ \\ and Mansoor Husain 1,2,3
}

\begin{abstract}
${ }^{1}$ Heart and Stroke Richard Lewar Centre of Excellence, University of Toronto, Toronto, Ontario, Canada
${ }^{2}$ Division of Cellular and Molecular Biology, The Toronto General Hospital Research Institute, Toronto, Ontario, Canada

${ }^{3}$ Department of Laboratory Medicine and Pathobiology, University of Toronto, Toronto, Ontario, Canada

${ }^{4}$ The Terrence Donnelly Heart Centre, St. Michael's Hospital, Toronto, Ontario, Canada

${ }^{5}$ Departments of Pediatrics and Pharmacology, University of Alberta, Edmonton, Alberta, Canada
\end{abstract}

Address correspondence to: Duncan J. Stewart, St. Michael's Hospital,

7-081 Queen, 30 Bond Street, Toronto, Ontario M5B 1W8, Canada.

Phone: (416) 864-5724; Fax: (416) 864-5914; E-mail: stewartd@smh.toronto.on.ca.

Duncan J. Stewart and Mansoor Husain contributed equally to this work.

Received for publication May 15, 2001, and accepted in revised form January 28, 2002.

\begin{abstract}
Increased inducible nitric oxide synthase (iNOS) expression is a component of the immune response and has been demonstrated in cardiomyocytes in septic shock, myocarditis, transplant rejection, ischemia, and dilated cardiomyopathy. To explore whether the consequences of such expression are adaptive or pathogenic, we have generated a transgenic mouse model conditionally targeting the expression of a human iNOS cDNA to myocardium. Chronic cardiac-specific upregulation of iNOS in transgenic mice led to increased production of peroxynitrite. This was associated with a mild inflammatory cell infiltrate, cardiac fibrosis, hypertrophy, and dilatation. While iNOS-overexpressing mice infrequently developed overt heart failure, they displayed a high incidence of sudden cardiac death due to bradyarrhythmia. This dramatic cardiac phenotype was rescued by specific attenuation of transgene activity. These data implicate cardiomyocyte iNOS overexpression as sufficient to cause cardiomyopathy, bradyarrhythmia, and sudden cardiac death.
\end{abstract}

J. Clin. Invest. 109:735-743 (2002). DOI:10.1172/JCI200213265.

\section{Introduction}

Nitric oxide (NO) is produced in vivo by a group of three NO synthases (NOSs): namely, the neuronal (nNOS), inducible (iNOS), and endothelial (eNOS) isoforms. Prerequisites for $\mathrm{NO}$ generation by NOS are L-arginine and oxygen, in addition to cofactors tetrahydrobiopterin $\left(\mathrm{BH}_{4}\right), \mathrm{NADPH}$, flavin adenine dinucleotide (FAD), flavin mononucleotide (FMN), heme, and calmodulin (1). Unlike constitutively expressed NOS isoforms (nNOS and eNOS), iNOS is regulated primarily at the transcriptional level. At baseline there is little, if any, detectable iNOS expression in any cell type. However, in response to inflammatory cytokines or endotoxins there is a robust upregulation of iNOS mRNA and protein in virtually every nucleated cell type (2). Induction of iNOS expression is mediated through cytokine-inducible transcription factors, such as IFN regulatory factor- 1 and NF- $\mathrm{KB}$ to elements within the iNOS promoter $(3,4)$. Because iNOS is irreversibly bound to calmodulin throughout the range of physiological $\mathrm{Ca}_{\mathrm{i}}^{2+}$, increased iNOS expression is usually associated with elevated NOS activity (5).

In conditions of $\mathrm{L}$-arginine or $\mathrm{BH}_{4}$ deficiency, $\mathrm{NOS}$ isoforms may reduce molecular oxygen to superoxide $\left(\mathrm{O}_{2}{ }^{-}\right)$, a reactive oxygen species $(6,7)$. Reactive nitrogen intermediates formed by the interaction of $\mathrm{NO}$ with $\mathrm{O}_{2}{ }^{-}$(e.g., peroxynitrite, $\mathrm{ONOO}^{-}$) and $\mathrm{O}_{2}^{-}$itself are toxic species capable of causing tissue damage (8). The simultaneous production of $\mathrm{NO}$ and $\mathrm{O}_{2}{ }^{-}$by activated immune cells has been implicated in controlling the spread of invading microbes, viruses, and cancerous cells.

Intracoronary $\mathrm{L}-\mathrm{N}^{\mathrm{G}}$-monomethyl arginine (L-NMMA) reveals a positive inotropic effect of NOS activity in normal myocardium (9). However, increased iNOS expression has been reported in pancreatic (10), intestinal (11), neuronal (12), and cardiac pathologies (13-16) and has been hypothesized to contribute to the progression of associated diseases (2). Consistent with a pathogenic role, iNOS knockout mice were less susceptible to LPS-induced septic shock (17) and had better outcomes after cardiac allograft transplantation, with a decrease in apoptosis $(18,19)$. Furthermore, the iNOS inhibitor S-methylisothiourea improved survival in rodent septic shock and rabbit myocardial infarction $(20,21)$. While these data suggest a causative or exacerbating role for iNOS in cardiac pathology, the specific contribution of increased iNOS expression and activity in isolation of the myriad other inflammatory mediators is unknown (22). We hypothesized that cardiac overexpression of iNOS would lead to a cardiotoxic phenotype, the ultimate pathogenesis depending, perhaps, on the balance between $\mathrm{NO}$ and $\mathrm{O}_{2}{ }^{-}$generation. 
In this study the $\alpha$-myosin heavy chain $(\alpha-\mathrm{MHC})$ promoter-dependent expression of a tetracycline-responsive transcriptional activator (tTA) (23) was used to conditionally modulate the cardiomyocyte overexpression of iNOS (Figure 1). This strategy takes advantage of tetracycline-modulated binding of tTA to the tTAresponsive element (24) of a bidirectional promoter $\left(\mathrm{pB}_{\mathrm{i}-1}\right)$. In the absence of tetracycline or its more watersoluble analogue doxycycline (DOX), tTA binds specifically to the $\mathrm{pB}_{\mathrm{i}-1}$ promoter, driving transcription of adjacent gene cassettes. DOX represses the interaction of the tTA protein with $\mathrm{pB}_{\mathrm{i}-1}$, allowing conditional suppression of downstream transgenes.

\section{Methods}

Transgenic mouse production. A full-length human iNOS cDNA was cloned into pBiGN (a derivative of $\mathrm{pBiG}$; CLONTECH Laboratories Inc., Palo Alto, California, USA), to create pBiGN:iNOS (Figure 1). Flanking bacterial sequences were removed and the transgene linearized with Not1. Pronuclei injections were performed by the Hospital for Sick Children transgenic facility (Toronto, Ontario, Canada) as described (25). Resulting progeny were screened for founders by PCR and Southern blot analyses using primers and probes for $\beta$-galactosidase ( $\beta$-gal) and human iNOS.

$R T-P C R$. Total RNA was extracted from organs using RNeasy (QIAGEN Inc., Valencia, California, USA), treated with DNAse1 and Super Script II reverse transcriptase per manufacturer's specifications (Invitrogen Life Technologies Inc., Burlington, Ontario, Canada). PCR was performed with human iNOS-specific primers (5'-TCT TGG GTC TCC GCT TCT CGT C-3'; 5'TGG CTG GTA CAT GGG CAC AGA G-3') and GAPDHspecific primers ( $5^{\prime}$-TCC ACC ACC CTG TTG CTG TAG- $3^{\prime}$; $5^{\prime}$-GAC CAC AGT CCA TGA CAT CAC T-3').

Western blot analysis. Hearts were homogenized in icecold $10 \mathrm{mM}$ Tris- $\mathrm{HCl}, 1 \mathrm{mM}$ EDTA, and protease inhibitors. Protein samples were run on a $4-12 \%$ gradient Tris-glycine gel and transferred to nitrocellulose. Membranes were incubated in blocking buffer: $3 \%$ milk in TBS (20 mM Tris-HCl, pH 7.4, 100 mM NaCl, 0.1\% Tween 20), then anti-iNOS Ab (1:333; Santa Cruz Biotechnology Inc., Santa Cruz, California, USA) for 1 hour at $21^{\circ} \mathrm{C}$, washed in TBS, and incubated with peroxidase-conjugated anti-rabbit secondary Ab (1:1,000; Sigma Chemical Co., St. Louis, Missouri, USA) for 1 hour. Immunoreactivity was detected with enhanced chemiluminescence (Amersham Biosciences, Baie D'Urfe, Quebec, Canada). $\beta$-galstaining. Frozen $6-\mu \mathrm{m}$ sections were fixed in cold acetone for 10 minutes, air-dried, washed in PBS, stained in filtered X-gal solution for 4 hours, washed, and counterstained with eosin as described (26).

Immunostaining. Frozen sections were thawed to $21^{\circ} \mathrm{C}$, washed in PBS, incubated in blocking buffer ( $5 \%$ goat serum in PBS), and then rabbit anti-iNOS polyclonal $\mathrm{Ab}$ (1:50; Transduction Laboratories, Lexington, Kentucky, USA) in $0.3 \%$ Triton X-100 in PBS for 1 hour at $21^{\circ} \mathrm{C}$. Following PBS washes, sections were treated with FITC-conjugated anti-rabbit Ab (1:250; PharMingen), washed again, counterstained with nuclear fast red (Sigma Chemical Co.), mounted, and examined with a confocal microscope.

For nitrotyrosine (NO-Y) immunostaining, tissues were harvested and fixed in 5\% formalin in PBS, embedded in paraffin, and cut to $8 \mu \mathrm{m}$. Sections were deparaffinized with xylene and then rehydrated. Sections were treated with $0.01 \%$ Triton in PBS for 5 minutes and washed in PBS. Background peroxidase was blocked with $3 \% \mathrm{H}_{2} \mathrm{O}_{2}$ in PBS for 15 minutes, followed by PBS wash. Sections were blocked with $10 \%$ goat serum, $3 \%$ BSA in PBS for 20 minutes, then incubated with rabbit polyclonal anti-NO-Y Ab (1:200; Upstate Biotechnology Inc., Lake Placid, New York, USA) in 5\% goat serum and $1 \% \mathrm{BSA}$ in $\mathrm{PBS}$ for 1 hour at $37^{\circ} \mathrm{C}$. After PBS wash, sections were treated with biotinylated anti-rabbit IgG (1:500; Jackson ImmunoResearch Laboratories Inc., West Grove, Pennsylvania, USA), washed again, and incubated with streptavidin-horseradish peroxidase conjugate (1:250; Jackson ImmunoResearch Laboratories Inc.) for 1 hour at $37^{\circ} \mathrm{C}$. Finally, sections were washed in PBS, incubated with diaminobenzidine (Sigma Chemical Co.), counterstained with methyl green, dehydrated, and mounted. NO-Y staining was scored on a scale $(0=$ none, $1=$ mild, $3=$ moderate, $5=$ severe) integrating the intensity and extent of peroxidase-positive cardiomyocyte staining by two independent observers blinded to the identity of the specimens.

Nitrate/nitrite measurement. The nitrate/nitrite colorimetric assay (Greiss reagent) was used to measure the levels of stable $\mathrm{NO}$ byproducts $\left(\mathrm{NO}_{x}\right)$ in urine samples, per manufacturer's protocols (Cayman Chemical, Ann Arbor, Michigan, USA). Plasma nitrate/nitrite was quantified using a NO sensor (Innovative Instruments Inc.,

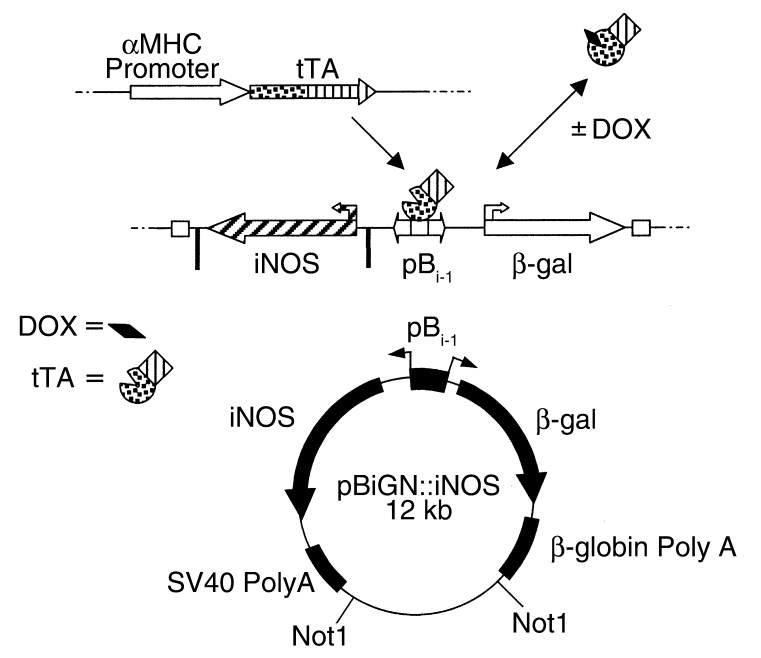

\section{Figure 1}

Diagrammatic representation of the binary tetracycline-regulated gene expression system and the construct used to produce iNOS $\beta$ gal transgenic mice. DOX, doxycycline; tTA, tetracycline-responsive transcriptional activator; $\mathrm{pB}_{\mathrm{i}-1}, \mathrm{tTA}$-responsive bidirectional promoter, $\beta$-gal, $\beta$-galactosidase. 
Tampa, Florida, USA), following reduction with excess $\mathrm{NaI} / 0.1 \mathrm{M} \mathrm{H}_{2} \mathrm{SO}_{4}$ and measurement of $\mathrm{NO}$ production. L-Citrulline formation assay. Heart tissue was ground to a fine powder in liquid $\mathrm{N}_{2}$ and homogenized in 4 vol. of buffer (10 mM HEPES, pH 7.2, 0.32 M sucrose, 0.1 $\mathrm{mM}$ EDTA, $1 \mathrm{mM}$ DTT, and protease inhibitors). The homogenate was centrifuged and aliquots of the supernatants were incubated for 60 minutes at $37^{\circ} \mathrm{C}$ with (a) assay cocktail: $50 \mathrm{mM}$ L-valine, $1 \mathrm{mM}$ DTT, $0.1 \mathrm{mM}$ NADPH, $0.1 \mathrm{mM} \mathrm{BH}_{4}, 1 \mathrm{mM}$ L-citrulline, $18 \mu \mathrm{M}$ L-arginine, $2 \mu \mathrm{M} \mathrm{L}-\left[{ }^{14} \mathrm{C}\right]$ arginine, $1 \mathrm{mM} \mathrm{MgCl}_{2}$, and $0.2 \mathrm{mM}$ $\mathrm{CaCl}_{2}$ in $50 \mathrm{mM} \mathrm{KH}_{2} \mathrm{PO}_{4}, \mathrm{pH} 7.2$; (b) cocktail plus 1 mM EGTA; or (c) cocktail plus $1 \mathrm{mM}$ EGTA plus $1 \mathrm{mM}$ L-NMMA, to determine the total and $\mathrm{Ca}^{2+}$-independent NOS activity. NOS activity was quantified by measuring $\mathrm{L}-\left[{ }^{14} \mathrm{C}\right]$ citrulline with a liquid scintillation counter, following removal of unreacted $\mathrm{L}-\left[{ }^{14} \mathrm{C}\right]$ arginine with cationic Dowex resin.

NADPH-diaphorase staining. Frozen sections were washed in $100 \mathrm{mM}$ Tris-HCl (pH 7.6) and incubated at $37^{\circ} \mathrm{C}$ for 30 minutes in $1 \mathrm{mM}$ NADPH, $0.2 \mathrm{mM}$ nitro blue tetrazolium, $0.2 \%$ Triton X-100 in $100 \mathrm{mM}$ Tris$\mathrm{HCl}$. Sections were washed again, counterstained with eosin, dehydrated, and sealed.

Atrioventricular node bistopathology and acetylcholineesterase activity. The Atrioventricular (AV) node region was identified in frozen, $10-\mu \mathrm{M}$ frontal-plane cardiac sections at the boundary of the interventricular and membranous interatrial septa. The identity of conduction tissue was verified with acetylcholine-esterase (AChE) staining (27). After washes in $0.1 \mathrm{M}$ sodium acetate, $\mathrm{pH}$ 6 , slides were incubated in AChE solution $(0.5 \mathrm{mg} / \mathrm{ml}$ acetylcholine iodide, $40 \mathrm{mM}$ sodium acetate, $2 \mathrm{mM}$ acetic acid, $5 \mathrm{mM}$ sodium citrate, $3 \mathrm{mM}$ cupric sulfate, $80 \mu \mathrm{m}$ tetraisopropyl pyrophosphoramide, $0.5 \mathrm{mM}$ potassium ferricyanide, $\mathrm{pH} 5.5$ ) for 2 hours at $37^{\circ} \mathrm{C}$. Sections were washed, treated with diaminobenzidine, washed again, dehydrated, and mounted.

Connexin-40 immunostaining. For connexin-40 (Cx40) immunostaining, $10-\mu \mathrm{m}$ frozen sections were fixed in cold acetone, air-dried, hydrated in PBS, incubated in blocking solution (10\% goat serum, 3\% BSA/PBS) for 1 hour, then in polyclonal $\mathrm{Cx} 40 \mathrm{Ab}$ (1:200; Chemicon International, Temecula, California, USA) in 5\% goat serum, 1\% BSA in PBS for 1 hour. Sections were then washed with PBS and incubated for 1 hour in FITCconjugated anti-rabbit Ab (1:200; Santa Cruz Biotechnology Inc.). Slides were washed again and mounted in $50 \%$ glycerol for fluorescence imaging.

Histological scoring of cardiac sections. A cardiac pathologist, blinded to the identity of the specimens, scored inflammatory cell infiltration, necrosis, fibrosis, and calcification in sections stained with hematoxylin and eosin (H\&E), Movat's stain, Mason's stain, and picrosirius red. Each parameter was given a value of $0-4$ on a published scale (28), for a maximum score of 16 .

Left ventricular hemodynamics. Animals were anesthetized using ketamine- $\mathrm{HCl}(100 \mathrm{mg} / \mathrm{kg}$ intraperitoneally) and xylazine- $\mathrm{HCl}(10 \mathrm{mg} / \mathrm{kg}$ intraperitoneally) and placed on a warming pad. The right common carotid artery was isolated following midline neck incision and cannulated using a Millar Mikro-tip pressure transducer $(1.4 \mathrm{~F}$ sensor, $2 \mathrm{~F}$ catheter). Hemodynamic traces were recorded in the common carotid and left ventricle.

Echocardiography. Mice were lightly anesthetized with ketamine- $\mathrm{HCl}$ (70 mg/kg intraperitoneally) and xylazine$\mathrm{HCl}(7 \mathrm{mg} / \mathrm{kg}$ intraperitoneally), and placed on a warming pad. M-mode echocardiography was performed by a blinded observer, using a Hewlett Packard 5500 ultrasound device and a $12-\mathrm{MHz}$ phased-array probe.

Electrocardiography. A dorsally mounted radio frequency transmitter and wire leads (lead II configuration) were implanted subcutaneously under anesthesia. Chronic electrocardiogram (ECG) recordings from conscious mice were acquired with the Data Sciences International telemetry system (Data Sciences International, St. Paul, Minnesota, USA). Acute ECG recordings were obtained in lightly anesthetized animals using subcutaneous platinum pins placed at the base of each limb and the sternum and a Gould recorder (Kent Scientific Corporation, Litchfield, Connecticut, USA). Approximately 10-second runs of each of leads I, II, III, aVr, aVL, and aVF were recorded.

All animal experimentation was conducted in accordance with standard operating protocols approved by the Toronto General Hospital Animal Care Committee.

\section{Results}

iNOS $\beta$ gal founder lines. Single cell pronuclei injections with pBiGN:iNOS produced four transgene positive founders (240i, 365iA, 365iB, and $365 \mathrm{iC}$ ) out of 30 live births. These founders transmitted the iNOS $\beta$ gal transgene to offspring and did not exhibit any developmental or reproductive abnormalities. One line (240i) was also bred to homozygosity $\left(\mathrm{iNOS}^{+/+}\right.$) without apparent defects for the exclusive purpose of increasing the generation of heterozygous iNOS ${ }^{+/-}$progeny in crosses with mice harboring the $\alpha \mathrm{MHC}$ promoter-driven tTA cassette ( $\alpha \mathrm{MtTA})$. In the absence of the $\alpha \mathrm{MtTA}$ transgene there was no human iNOS expression in $\mathrm{iNOS}^{+/-}$or $\mathrm{iNOS}^{+/+}$mice.

$\beta$-gal and $i N O S$ expression. Heterozygous or homozygous iNOS $\beta$ gal mice were crossed with $\alpha \mathrm{MtTA}$ mice, and the expressions of tTA-responsive transgenes were examined. Crossing iNOS ${ }^{+}$with $\alpha \mathrm{MtTA}^{+}$mice resulted in significantly fewer iNOS ${ }^{+} / \alpha \mathrm{MtTA}^{+}$progeny than expected (17 of $110=15 \%$ vs. $25 \%$ expected, or 16 of $62=26 \%$ for mothers treated with DOX, $P<0.05)$, indicating that iNOS overexpression during embryonic development was associated with significant fetal loss. While this observation is of interest, future studies are planned to explore the mechanisms underlying developmental toxicity, and the present study has focused primarily on the consequences of cardiac iNOS overexpression in the postnatal period. Accordingly, all maternal mice were treated with DOX to suppress transgene expression during fetal development. This suppression was released by DOX removal in the postnatal period to study effects of cardiac iNOS overexpression in adult myocardium. $\beta$-gal staining was done 

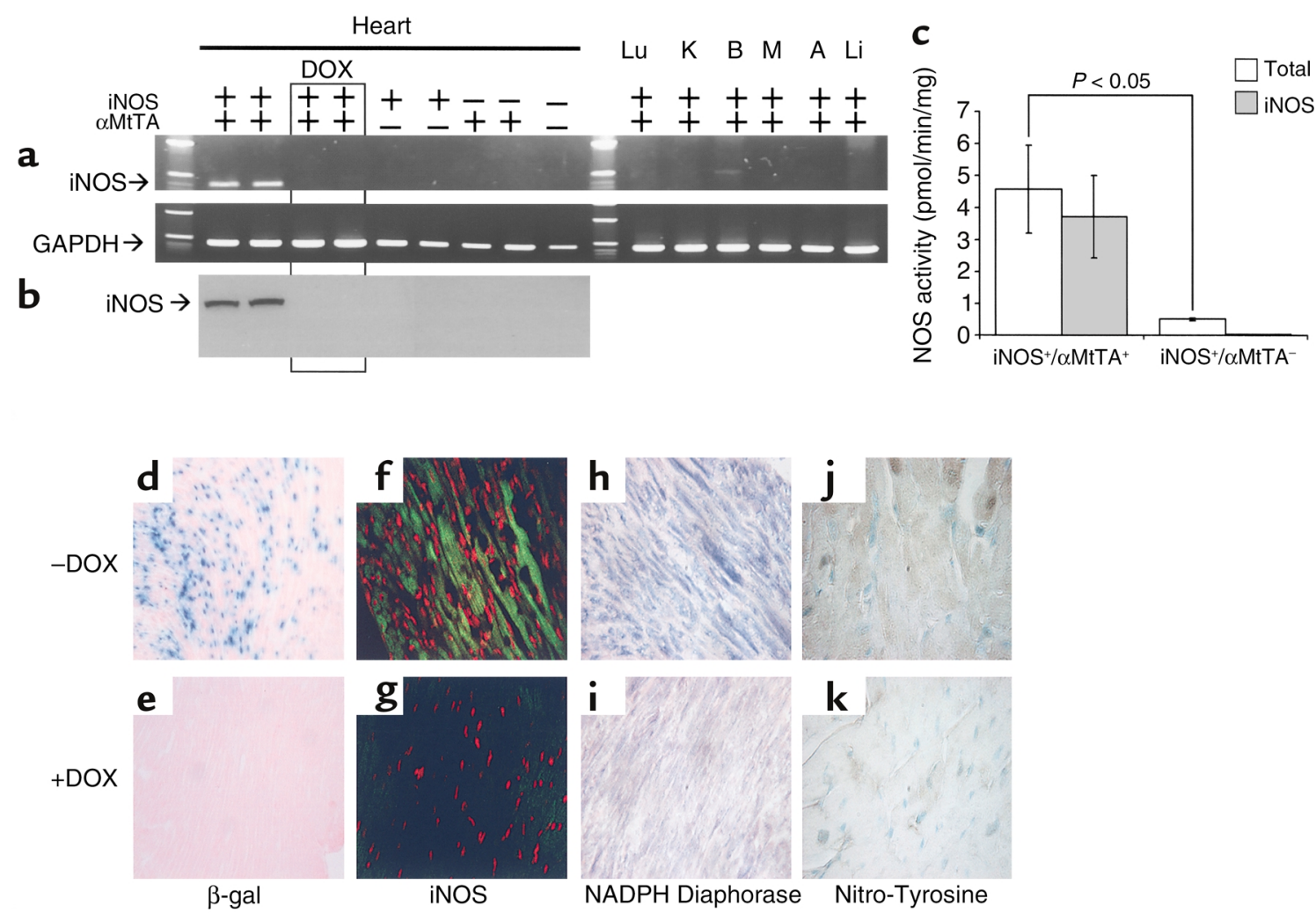

\section{Figure 2}

( $\mathbf{a}$ and $\mathbf{b}$ ) Expression of human iNOS mRNA (RT-PCR) and iNOS protein (Western blot) in hearts of iNOS ${ }^{+} / \alpha \mathrm{MtTA}^{+} \mathrm{mice}$ in the presence and absence of DOX $(200 \mu \mathrm{g} / \mathrm{ml}$ in drinking water) and nonbinary littermate controls. (c) Calcium-independent (iNOS) and total NOS activities in cardiac extracts of iNOS ${ }^{+} / \alpha \mathrm{MtTA}^{+}$mice and nonbinary littermate controls, $n=5$. ( $\mathbf{d}$ and $\left.\mathbf{e}\right) \beta$-gal (blue staining), (f and $\mathbf{g}$ ) iNOS (green immunofluorescence), ( $\mathbf{h}$ and $\mathbf{i}$ ) NADPH-diaphorase (dark blue staining), and ( $\mathbf{j}$ and $\mathbf{k}$ ) NO-Y (brown staining) of cardiac sections from $\mathrm{iNOS}^{+} / \alpha \mathrm{MtTA}^{+}$mice in the presence $(+)$and absence $(-)$of DOX.

to assess nuclear-localized $\beta$-gal reporter activity in aorta, brain, kidney, liver, lung, spleen, skeletal muscle, and heart of $\mathrm{iNOS}^{+} / \alpha \mathrm{MtTA}^{+}$mice, in the presence and absence of DOX and in nonbinary littermates. $\beta$-gal activity was noted only in cardiac myocytes of $\mathrm{iNOS}^{+} / \alpha \mathrm{MtTA}^{+}$ mice from three of the four lines $(240 \mathrm{i}, 365 \mathrm{iB}$, and $365 \mathrm{iC})$, which was reduced to undetectable levels by DOX treatment $(200 \mu \mathrm{g} / \mathrm{ml}$ in drinking water) (Figure 2, $\mathrm{d}$ and e).

To measure transgenic iNOS expression, total cardiac cellular RNA was subjected to RT-PCR for human-specific iNOS and GAPDH control. Human-specific iNOS mRNA was detected in hearts of two of the remaining three lines (240i and 365iB) in the absence of DOX, but not nonbinary littermates (iNOS $-\alpha \mathrm{MtTA}^{+}$, $\mathrm{iNOS}^{+} / \alpha \mathrm{MtTA}^{-}, \mathrm{iNOS}^{-} / \alpha \mathrm{MtTA}^{-}$) or DOX-treated binary controls (Figure 2a, $n \geq 5$ for each group). No human iNOS mRNA was detected in any other organs of $\mathrm{iNOS}^{+} / \alpha \mathrm{MtTA}^{+}$mice.

Total cytosolic proteins extracted from hearts of $240 \mathrm{i}$ and $365 \mathrm{iB} \mathrm{iNOS}{ }^{+} / \alpha \mathrm{MtTA}^{+}$mice and control littermates, were used for Western blot with an iNOS-specific polyclonal Ab (Figure $2 \mathrm{~b}$ ). Binary iNOS ${ }^{+} / \alpha \mathrm{MtTA}^{+}$mice displayed expression of iNOS protein $(135 \mathrm{kDa})$, which was diminished to undetectable levels with DOX treatment. Cardiac iNOS protein expression was not observed in nonbinary littermate controls ( $n \geq 5$ for each group).
To determine the cellular localization of iNOS protein in the hearts of $\mathrm{NOS}^{+} / \alpha \mathrm{MtTA}^{+}$mice, immunostaining was performed. Figure 2, $\mathrm{f}-\mathrm{g}$, shows confocal images of cardiac sections from iNOS $/ \alpha \mathrm{MtTA}^{+}$mice in the (Figure 2f) absence and (Figure 2g) presence of DOX; iNOS protein expression is shown by green immunofluorescence. Note that iNOS immunoreactivity is localized to cytoplasm throughout the myocardium and is absent in the DOX-treated iNOS ${ }^{+} / \alpha \mathrm{MtTA}^{+}$control. These data demonstrate the conditional and myocardium-specific localization of $\beta$-gal activity and transgenic iNOS in binary iNOS ${ }^{+} / \alpha \mathrm{MtTA}^{+}$mice.

NOS activity: L-citrulline production assay, NADPHdiaphorase staining. To quantify levels of cardiac NOS activity, we measured the conversion of ${ }^{14} \mathrm{C}$-labeled $\mathrm{L}$-arginine to L-citrulline. Binary transgenic mice exhibited robust iNOS activity compared with control mice, which were not different from background $(P<0.05$, Figure 2c), and a tenfold increase in total NOS activity over nonbinary transgenic controls $(4.6 \pm 1.4$ vs. $0.5 \pm 0.1 \mathrm{pmol} / \mathrm{min} / \mathrm{mg}, n=5, P<0.05)$.

$\mathrm{NADPH}$-dependent diaphorase staining was used as a measure of in situ NOS activity in cardiac tissue sections (Figure $2, \mathrm{~h}$ and i). Binary iNOS ${ }^{+} / \alpha \mathrm{MtTA}^{+}$mice displayed increased diaphorase activity (dark blue staining) in myocardium, but not any other organ, in 


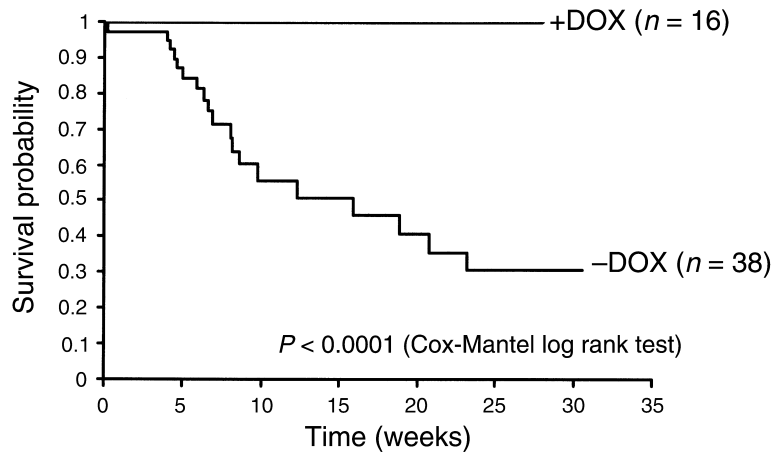

Figure 3

Kaplan-Meier analysis of survival in $\mathrm{iNOS}^{+} / \alpha \mathrm{MtTA}^{+}$mice in the presence and absence of DOX.

comparison with DOX-treated or nonbinary transgenic controls. These data demonstrate the conditional myocardium-specific increase in transgene-derived NOS activity in iNOS ${ }^{+} / \alpha \mathrm{MtTA}^{+}$mice.

NO production: urinary and plasma nitrites/nitrates and NO-Y staining. Measurements of urinary and plasma nitrite/nitrate $\left(\mathrm{NO}_{\mathrm{x}}^{-}\right)$, stable $\mathrm{NO}$ end products, and NO-Y immunostaining, a marker of peroxynitrite production, were used to determine NO and NO-Y levels, respectively, in iNOS ${ }^{+} / \alpha \mathrm{MtTA}^{+}$mice. $\mathrm{NO}_{x}{ }^{-}$levels in urine or plasma were not significantly different in $\mathrm{iNOS}^{+} / \alpha \mathrm{MtTA}^{+}$mice versus littermate controls (data not shown). The NO-Y staining score was significantly elevated in cardiac myocytes of $\mathrm{iNOS}^{+} / \alpha \mathrm{MtTA}^{+}$mice $(3.7 \pm 0.3)$ versus littermate control $(0.3 \pm 0.6, P<0.01$, $n=3$ ) (Figure 2, $\mathrm{j}$ and $\mathrm{k}$ ).

Phenotype of binary transgenic $i \mathrm{NOS}^{+} / \alpha M t \mathrm{TA}^{+}$mice. Binary transgenic $\mathrm{iNOS}^{+} / \alpha \mathrm{MtTA}^{+}$mice exhibited a significant in-crease in mortality as compared with iNOS ${ }^{+} / \alpha \mathrm{MtTA}^{+}$mice treated with DOX (Figure 3; Kaplan-Meier analysis, $P<0.0001)$. Postmortem examinations of $\mathrm{iNOS}^{+} / \alpha \mathrm{MtTA}^{+}$mice typically revealed normal cardiac morphology. However, a few deceased animals exhibited gross ventricular dilation and hypertrophy.

M-mode echocardiography was used to measure cardiac dimensions in $\mathrm{iNOS}^{+} / \alpha \mathrm{MtTA}^{+}$mice and littermate controls (Figure 4a). All animals

\section{Figure 4}

(a) LVEDD measured by M-mode echocardiography in $\mathrm{iNOS}^{+} / \alpha \mathrm{MtTA}^{+}$mice and littermate controls. Animals were fed DOX $(200 \mu \mathrm{g} / \mathrm{ml}$ in drinking water $)$ to suppress transgene expression and released at 10 weeks of age (day 0$)$. (b) Heart/body weight ratio in $\mathrm{iNOS}^{+} / \alpha \mathrm{MtTA}^{+}$mice $(n=3)$ and iNOS ${ }^{+} / \alpha \mathrm{MtTA}^{-}$lit- $\mathrm{iNOS}^{+} / \alpha \mathrm{MtTA}^{-}$ termates $(n=4)$. (c) H\&E, picro-sirius red, and Movat's staining of serial cardiac sections from an $\mathrm{iNOS}^{+} / \alpha \mathrm{MtTA}^{+}$mouse and control littermate. received DOX from conception until 10 weeks of age. DOX was then removed, and echocardiography was performed 0 and 30 days thereafter. At day 0 there was no difference in any left ventricular (LV) dimension (wall thickness or chamber size) in iNOS ${ }^{+} / \alpha \mathrm{MtTA}^{+}$mice versus controls. However, at 30 days there was a significant increase in the LV end-diastolic dimension (LVEDD) of the $\mathrm{iNOS}^{+} / \alpha \mathrm{MtTA}^{+}$group and no change in the nonbinary transgenic group. To explore if cardiac enlargement was associated with an increase in cardiac mass, heart-to-body weight ratios were measured in male and female iNOS ${ }^{+} / \alpha \mathrm{MtTA}^{+}$mice and age- and sex-matched littermate controls (Figure $4 \mathrm{~b}$ ). The heart-to-body weight ratio was significantly larger in $\mathrm{iNOS}^{+} / \alpha \mathrm{MtTA}^{+}$ mice as compared with control littermates. Together, these data indicate that increased iNOS activity leads to an increase in heart size and mass.

To determine if changes in LV function correlated with iNOS overexpression, $\mathrm{LV}$ hemodynamics were measured in 8- to 12-week-old iNOS ${ }^{+} / \alpha \mathrm{MtTA}^{+}$mice and age-, weight-, and sex-matched littermate controls in the absence of DOX. There was no significant difference in peak LV systolic pressure (105 \pm 6 vs. $96 \pm 4 \mathrm{mmHg}$, $P=0.22)$, LV end diastolic pressure ( $14 \pm 2$ vs. $16 \pm 2$ $\mathrm{mmHg}, P=0.55$ ) and $-\mathrm{dP} / \mathrm{dT}$ (first derivative of LV pressure over time) $(5,866 \pm 674$ vs. $4,988 \pm 370, P=0.23)$; however there was an increase in $+\mathrm{dP} / \mathrm{dT}(7,944 \pm 780 \mathrm{vs}$. $5,775 \pm 411, P=0.01)$ in iNOS ${ }^{+} / \alpha \mathrm{MtTA}^{+}(n=15)$ versus nonbinary transgenic mice $(n=35)$. These data suggest that iNOS overexpression did not exert negative effects on cardiac hemodynamics in the absence of heart failure.

Cardiac histology in iNOS ${ }^{+} / \alpha \mathrm{MtTA}^{+}$mice was also assessed by $\mathrm{H} \& \mathrm{E}$, picro-sirius red, and Movat's staining a

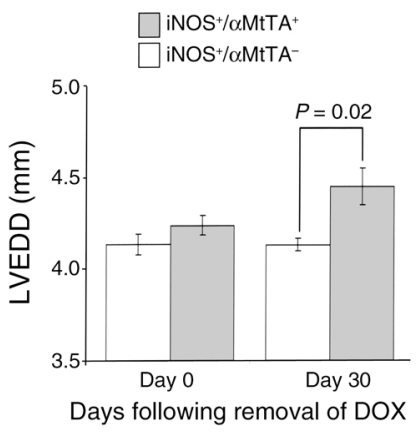

C

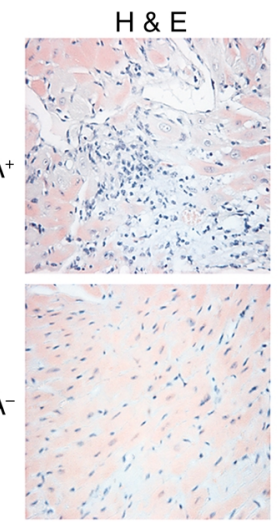

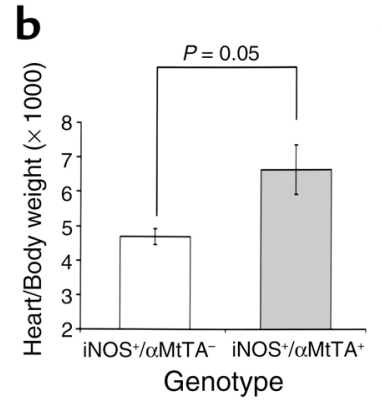

Picro-Sirius red

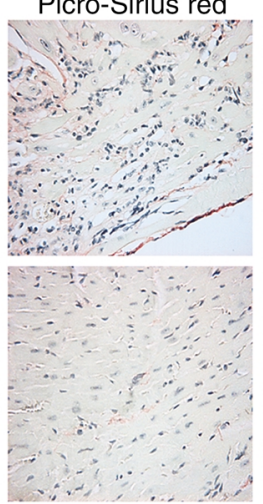

Movat's

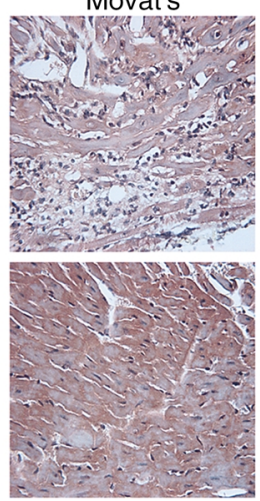


a

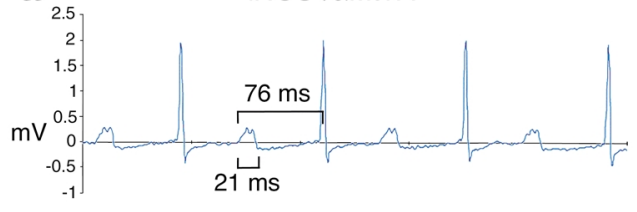

b

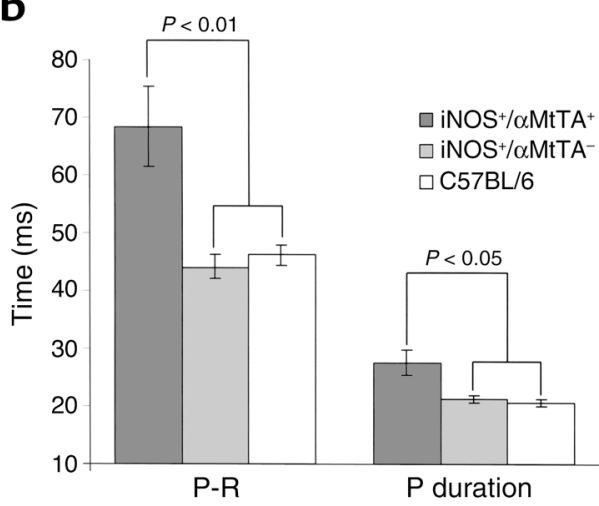

iNOS $^{+} / \alpha \mathrm{MtTA}$
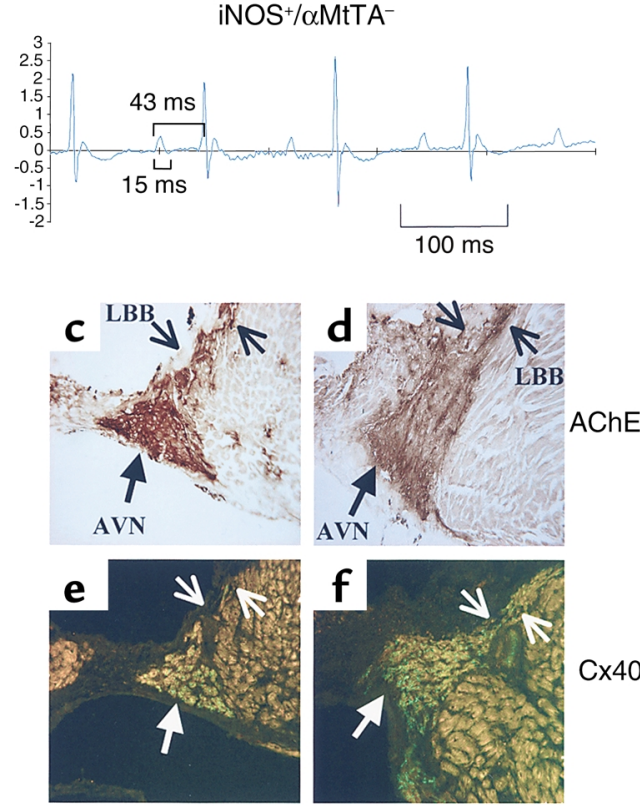

iNOS $/ \alpha \mathrm{MtTA}^{+}$ With heart block

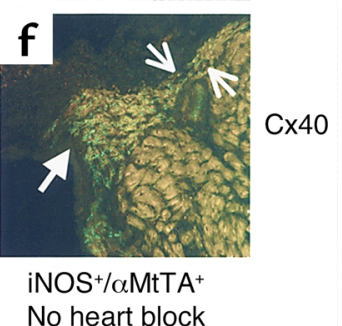

Figure 5

(a) Baseline ECG recordings (lead II) from conscious $\mathrm{iNOS}^{+} / \alpha \mathrm{MtTA}^{+}$and $\mathrm{iNOS}^{+} /$ $\alpha M t T A^{-}$control mice. (b) P-R interval and $\mathrm{P}$-wave duration from iNOS ${ }^{+} / \alpha \mathrm{MtTA}^{+}$mice, littermate controls, and wildtype C57BI/ 6 mice. (c and d) AChE (brown staining) and (e and f) $\mathrm{C} \times 40$ (green fluorescence) staining in adjacent serial sections from an $\mathrm{iNOS}^{+} / \alpha \mathrm{MtTA}^{+}$mouse with and one without heart block. LBB, left bundle branch; AVN, $A V$ node.
(Figure 4c). Ventricular myocardium of iNOS $^{+} /$ $\alpha \mathrm{MtTA}^{+}$mice $(n=6)$ displayed a mild increase in scores of inflammatory cell infiltration $(1.8 \pm 0.4)$ and myofibril necrosis $(1.6 \pm 0.5)$, but no evidence of calcification (0 of 4 ) or fibrosis (0 of 4). Corresponding scores in control littermates $(n=4)$ were 0 of 4 in each of these categories $(P<0.05)$.

Of note, a minority of $\mathrm{iNOS}^{+} / \alpha_{\mathrm{MtTA}}{ }^{+}$animals ( 6 of 78 mice) exhibited a phenotype that was clearly indicative of overt congestive heart failure later in life (15-30 weeks). These animals displayed an increased body weight, generalized edema, dyspnea, and lethargy. In addition, LV studies in these mice demonstrated significantly depressed hemodynamics, while histopathology displayed increased chamber sizes and significant increases in interstitial collagen staining (data not shown).

Mechanism of sudden death in iNOS ${ }^{+} / \alpha M t T A^{+}$mice. To explore the mechanism of sudden death, ECG tracings were recorded acutely from 8 -week-old mice. Binary $\mathrm{iNOS}^{+} / \alpha \mathrm{MtTA}^{+}$mice displayed prolongation of the $\mathrm{P}-\mathrm{R}$ interval and $\mathrm{P}$-wave duration (Figure 5), leading to progressive heart block (Figure 6).

Of the four iNOS ${ }^{+} / \alpha \mathrm{MtTA}^{+}$mice monitored with chronic telemetry (Figure 6a), all developed second degree (Mobitz type 1) AV block with Wenckebach periodicity, i.e., progressive elongation of the P-R interval followed by dropped ventricular beats. These mice also had bifid P-waves, which in concert with the elongation in P-wave duration suggest atrial enlargement. Infrequently, 5- to 15-second bursts of ventricular tachycardia and up to eight consecutive dropped beats were also noted. More advanced (Mobitz type II and third degree) heart block was also noted in three of four animals, including sustained 2:1 heart block, AV dissociation, and idioventricular escape complexes. ECG recordings from DOX-treated iNOS ${ }^{+} / \alpha \mathrm{MtTA}^{+}$and nonbinary littermates did not display any of the irregularities listed above. Figure $6 \mathrm{~b}$ is an ECG telemetry recording of an occurrence of sudden cardiac death in an $\mathrm{iNOS}^{+} / \alpha \mathrm{MtTA}^{+}$mouse. In this animal, cardiac asystole was preceded by a short burst of 2:1 block (not shown).

$A V$ node histology and AchE activity, CX40 expression, and $N O-Y$ staining. Histology of the AV node was scored in $\mathrm{iNOS}^{+} / \alpha \mathrm{MtTA}^{+}$and littermate controls using Mason's, $\mathrm{H} \& \mathrm{E}$, and picro-sirius red staining. While a trend toward an increased inflammatory cell score was noted in the AV node of $\mathrm{iNOS}^{+} / \alpha \mathrm{MtTA}^{+}$mice in comparison with controls $(2.0 \pm 0.8$ vs. $0.8 \pm 0.8, n \geq 3, P=0.1)$, there was no difference in the fibrosis score $(1.9 \pm 0.3$ vs. $2.0 \pm 0.9, n \geq 3$, $P=0.9)$ and no evidence of calcification or necrosis.

AchE and Cx40 staining, markers of conductive tissue in the heart, were also examined in $\mathrm{iNOS}^{+} / \alpha \mathrm{MtTA}^{+}$ animals with $(n=2)$ and without $(n=2)$ evidence of heart block and littermate controls $(n=4)$. No significant difference in the pattern of AchE staining was noted in binary transgenic versus control mice, or in $\mathrm{iNOS}^{+} / \alpha \mathrm{MtTA}^{+}$mice with or without heart block (Figure $5, \mathrm{c}$ and $\mathrm{d}$ ). We confirmed previous reports of cardiac $\mathrm{Cx} 40$ expression being restricted to the atrial myocardium, AV node, and bundle branches, with low levels being expressed in the ventricular Purkinje network (29). However, we noted also an appreciable decrease in $\mathrm{Cx} 40$ immunostaining in the AV node, His, and bundle branch cells of $\mathrm{NOS}^{+} / \alpha \mathrm{MtTA}^{+}$mice with heart block as compared with iNOS ${ }^{+} / \alpha \mathrm{MtTA}^{+}$mice without heart block (Figure 5 , e and $\mathrm{f}$ ).

To determine if the presence of heart block and/or AV node histological changes were due to a localized increase in the products of iNOS activity, we compared NO-Ydetermined $\mathrm{ONOO}^{-}$production in the conduction 
tissues of $\mathrm{iNOS}^{+} / \alpha \mathrm{MtTA}^{+}$mice with $(n=2)$ and without $(n=2)$ evidence of advanced heart block. No appreciable increase in NO-Y staining was noted in the AV node versus other ventricular segments or in asymptomatic mice versus those with heart block (data not shown).

\section{Discussion}

Increased iNOS expression has been documented in biopsy and autopsy specimens from individuals with cardiac disease. To better understand the role of this protein in pathogenesis, we designed a transgenic model that targeted expression of iNOS to myocardium and could be regulated by the tetracycline conditional expression system. These mice displayed robust DOX-regulated cardiac-specific overexpression of iNOS activity with increased cardiomyocyte NO-Y staining indicative of elevated $\mathrm{ONOO}^{-}$generation. There were also increases in L-NAME-inhibited lucigenin chemiluminescence and in situ hydroethidine staining (data not shown), indices of $\mathrm{O}_{2}^{-}$production, but no evidence of increased levels of $\mathrm{NO}_{\mathrm{x}}{ }^{-}$in urine and plasma. Taken together, these data suggest iNOS-derived superoxide-dependent peroxynitrite generation. However, these findings are preliminary and require unequivocal confirmation of iNOS-dependent superoxide generation in vivo.

The generation of $\mathrm{O}_{2}{ }^{-}$by iNOS in vitro has been documented previously and occurs when there are limiting levels of $\mathrm{L}$-arginine or $\mathrm{BH}_{4}$ causing uncoupling of the reductase and oxidase domains of $\operatorname{NOS}(6,7)$. In conjunction with cytokine-induced iNOS upregulation, increased $\mathrm{L}$-arginine uptake and $\mathrm{BH}_{4}$ synthesis has been reported, facilitating the production of NO (30). However, due to $\mathrm{Ca}^{2+} /$ calmodulin independence, iNOS is constitutively active, leading to substrate depletion and a switch to $\mathrm{O}_{2}-$ production (7). This may be the case in our model, where there is an increase in myocardial NO-Y staining, a stable cellular byproduct of $\mathrm{ONOO}^{-}$, but no detectable change in $\mathrm{NO}_{\mathrm{x}}{ }^{-}$production. However, the necessary in vivo experiments to confirm iNOSdependent superoxide generation have yet to be performed.

\section{Figure 6}

(a) Progressive heart block in iNOS $^{+} / \alpha \mathrm{MtTA}^{+}$mice. Shown are five nonconsecutive 10-second ECG recordings (lead II) from conscious iNOS $^{+} / \alpha \mathrm{MtTA}^{+}$animals. P-R intervals are shown above each complex. Progressive $\mathrm{P}-\mathrm{R}$ elongation with a dropped beat is consistent with Wenkebach periodicity. (b) Cardiac arrest in an iNOS $^{+} / \alpha \mathrm{MtTA}^{+}$mouse. (c) Normal baseline ECG recordings from a littermate control mouse.

\section{a $\mathrm{iNOS}^{+} / \alpha \mathrm{MtTA}^{+}$}

Histological analysis of hearts from a group of iNOSoverexpressing mice displayed mild increases in inflammatory cell infiltration and myofibril necrosis, and no evidence of calcification or fibrosis. In comparison with a virus-induced model of myocarditis (28), the inflammatory phenotype of our model is very mild.

In myocardial ischemia-reperfusion injury, $\mathrm{O}_{2}{ }^{-}$produced by activated neutrophils can lead to activation of the complement pathway (31). Complement activation causes endothelial dysfunction and facilitates the adhesion and further recruitment of circulating immune cells (32). In the ischemia-reperfusion model of increased myocardial $\mathrm{O}_{2}{ }^{-}$generation, upregulation of platelet-activating factor and subsequent formation of membrane attack complexes and cellular necrosis has also been observed (31). It is tempting to speculate that similar mechanisms may underlie the cellular infiltrates and myocardial damage noted in our model.

Ventricular size and heart-to-body weight ratio was significantly increased in binary transgenic mice compared with littermate controls. These data clearly suggest that increased myocardial iNOS activity is capable of initiating a process of cardiac remodeling that is characterized by ventricular dilatation and hypertrophy. However, it is worth noting that these relatively modest structural changes were unlikely to have contributed to the remarkably high incidence of sudden death. Indeed, many animals suffering sudden death displayed near normal postmortem cardiac pathology.

ECG recordings revealed a significant increase in the $\mathrm{P}$-wave duration and P-R interval in binary transgenic mice compared with control mice. While the development of bifid P-waves further suggests atrial enlargement, the above changes are also consistent with prolonged a

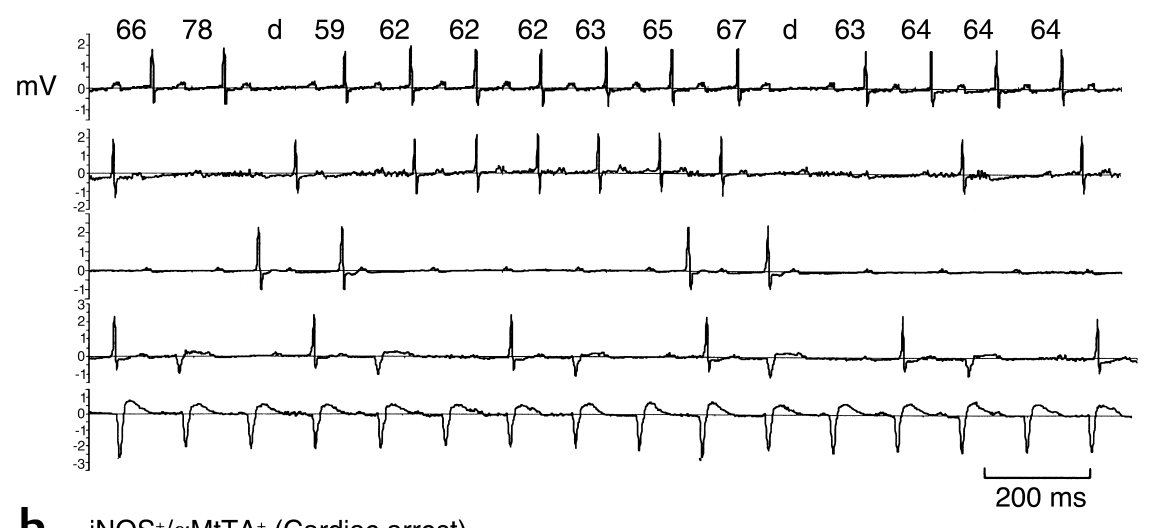

b $\mathrm{iNOS}^{+} / \alpha \mathrm{MtTA}^{+}$(Cardiac arrest)
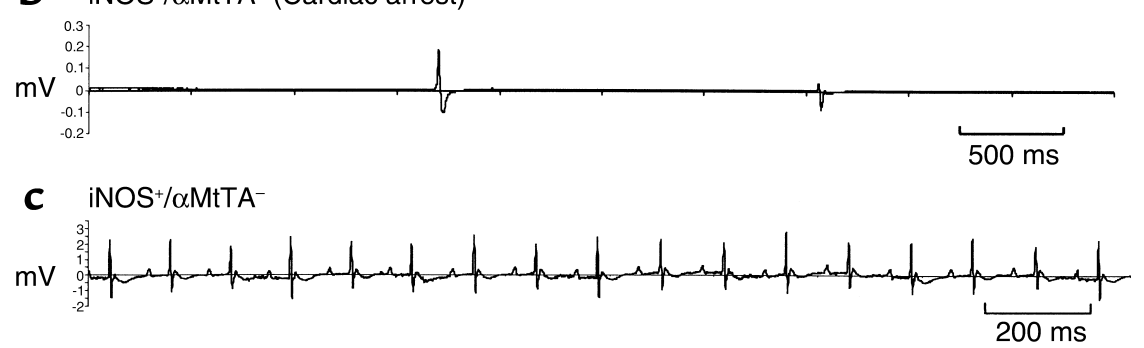
trial and/or AV nodal conduction. Importantly, the significant mortality of iNOS-overexpressing mice correlated directly with these conduction abnormalities. Chronic ECG telemetry showed a stepwise progression of heart block ultimately leading to asystole. Taken together, the above data link an increase in cardiac iNOS activity in the mouse, with progressive defects in cardiac conduction and the development of lethal bradyarrhythmias.

These data are intriguing in light of the dystrophinutrophin double-knockout transgenic mouse. This model displays a muscular dystrophy phenotype, with cardiac arrhythmias and cardiomyopathy (33). Because dystrophin is known to associate with nNOS (34), the decrease in cardiac nNOS expression observed in this knockout model was to be expected. However, these mice also exhibit a sevenfold increase in cardiac iNOS expression (33). While changes in overall NOS activity and/or the presence of cardiac $\mathrm{O}_{2}{ }^{-}$or NO-Y has not been examined in these knockout mice, the propensity for arrhythmia parallels that of our transgenic model. Similarly, increased cardiac $\mathrm{O}_{2}^{-}$production in models of myocardial ischemia-reperfusion injury, has also been correlated with a propensity for arrhythmia. Treatments with $\mathrm{O}_{2}{ }^{-}$donors lead to an increased frequency of cardiac arrhythmias (35), while $\mathrm{O}_{2}{ }^{-}$scavengers reduce arrhythmias in cardiac ischemia-reperfusion models (36).

$\mathrm{AV}$-node histology revealed a mild increase in inflammatory cell infiltration in binary transgenic mice, with no difference in necrosis, fibrosis, or calcification. Although the fibrosis score of the AV node in binary transgenic animals $(1.9 \pm 0.3)$ appears greater than that of the ventricular myocardium (0.0), the same difference is noted in littermate controls $(2.0 \pm 0.9$ vs. 0.0$)$. We believe that these data reflect the normally increased collagen composition of the atrioventricular septum rather than pathological fibrosis.

While AChE activity was not appreciably different in iNOS ${ }^{+} / \alpha \mathrm{MtTA}^{+}$mice with electrical dysfunction as compared with controls, the expression of $\mathrm{Cx} 40$ was clearly reduced in conduction system cells of $\mathrm{iNOS}^{+} / \alpha \mathrm{MtTA}^{+}$mice with evidence of heart block. These findings are intriguing in that they are the first demonstration of a postnatal downregulation of $\mathrm{Cx} 40$ expression. Indeed, the electrical phenotype produced by the release from DOX-mediated suppression of iNOS in adult iNOS ${ }^{+} / \alpha \mathrm{MtTA}^{+}$mice is similar to that seen in mice deficient in $\mathrm{Cx} 40$ (37) and other transgenic models with impaired conduction system development and depressed levels of $\mathrm{Cx} 40(29,38)$. This implicates the downregulation of cardiac $\mathrm{Cx} 40$ in the pathophysiology of adult conduction disease.

While depressed $\mathrm{Cx} 40$ expression may contribute to the cardiac electrical dysfunction of $\mathrm{iNOS}^{+} / \alpha \mathrm{MtTA}^{+}$ mice, the increase in inflammatory cells within the AV node, as well as the elevation in $\mathrm{ONOO}^{-}$and/or $\mathrm{O}_{2}{ }^{-}$ production, may result in other functional defects yet to be defined. Future studies aimed at specific inhibition of $\mathrm{O}_{2}^{-}$production, for example through crosses with oxidative stress prevention-overexpressing (SOD- overexpressing) mice, may inform directly on the relative contribution of these possible mechanisms.

Importantly, a few iNOS-overexpressing mice $(<8 \%)$ developed overt heart failure with depressed cardiac hemodynamic function, gross structural cardiomyocyte damage, and significant increases in interstitial collagen, ventricular thickness, and chamber size. However, increased cardiomyocyte iNOS activity did not cause a decrease in cardiac function before the development of overt heart failure. Rather, in the early stages of our model, binary transgenic mice displayed significantly increased contractility. This finding suggests that increased iNOS activity may initially positively influence cardiac contractility, consistent with human experiments in which intracoronary NOS inhibition decreased contractility in nonfailing myocardium (9). However, we cannot rule out the possibility that this may also be explained by an increased PR interval, with a preloaddependent increase in contractility. The fact that mice that developed overt heart failure tended to be older animals that had avoided sudden death, suggests that iNOSmediated heart failure is a pathology requiring a longer timeframe than that causing arrhythmia.

In contrast to our results, Heger et al. recently report that increased cardiac iNOS activity did not alter cardiac structure or function (39). However, this apparent difference in phenotype likely reflects a critical difference in experimental design, most importantly our use of a conditional system for cardiac-selective transgene expression. Given our clear demonstration of developmental lethality in the absence of DOX, we hypothesize that the nonconditional direct transgene approach employed by Heger et al. likely resulted in negative selection against lines with pathological levels of iNOS activity. Indeed, only S-ethylisothiourea-inhibitable iNOS activity was measured in the Heger animals, and these data are presented as a fold increase over control mice exhibiting no significant iNOS activity over baseline (39). Unfortunately, such a comparison is prone to overestimation of the absolute levels of iNOS activity and may have little bearing on total NOS activity. For this reason, we reported the increase in total NOS activity in the present study, with binary transgenic animals exhibiting greater than tenfold increase over nonbinary littermates. Because Heger et al. did not present total NOS activity, a comparison with our model is difficult. Additional variables influencing the difference in phenotype with the nonconditional model may include epigenetic transgene dosing effects or adaptive compensatory effects of other loci such as the upregulation of genes regulating substrate (L-arginine) or cofactor $\left(\mathrm{BH}_{4}\right)$ availability or oxidative stress prevention (SOD).

In conclusion, we believe that the role of iNOS in cardiac pathophysiology is complex. The present transgenic model suggests that the specific activity of this protein, in isolation of the myriad of other components of the immune response, is sufficient to produce measurable changes in cardiac structure and function. This model will allow further exploration of the effects of iNOS- 
derived $\mathrm{NO}, \mathrm{O}_{2}^{-}$, or $\mathrm{ONOO}^{-}$production by manipulating the availability of critical substrates (e.g., supplementation with $\mathrm{L}$-arginine or $\mathrm{BH}_{4}$ ). The data from this study suggest there is a correlation between chronic overexpression of iNOS and $\mathrm{ONOO}^{-}$generation with cardiac enlargement, conduction defects, sudden cardiac death, and, less commonly, heart failure. The conditional nature of the system allows design of highly specific studies aimed at examining the timing and reversibility of cardiac sequelae associated with increased myocardial iNOS activity. Finally, the finding that increased cardiac iNOS activity has pathogenic potential suggests that selective inhibition of elevated iNOS activity may represent a valuable strategy for treatment of a variety of human cardiovascular conditions.

\section{Acknowledgments}

The authors thank Fred Keeley, Myron Cybulsky, Phillip Marsden, and Sukriti Nag. I.N. Mungrue is supported by a Canadian Institutes of Health Research/Heart and Stroke Foundation of Ontario (CIHR/HSFO) studentship and a Heart and Stroke Richard Lewar Centre of Excellence Lorne Phenix Award. R. Gros is supported by an HSFO Fellowship and the Edward Christie Stevens and Evelyn McGloin Awards. X. You was supported by a Medical Research Council of Canada/Canadian Hypertension Society Fellowship. T. Csont is an Alberta Heritage Foundation for Medical Research (AHFMR) Fellow. R. Schulz is an AHFMR Senior Scholar and is supported by CIHR operating grant MT-11563. This study was supported by CIHR operating grants MT-11620 (D.J. Stewart) and MT-14648 (M. Husain) and HSFO operating grants NA-4789 (D.J. Stewart) and NA-4389 (M. Husain). M. Husain is a CIHR Clinician Scientist.

1. Knowles, R.G., and Moncada, S. 1994. Nitric oxide synthases in mammals. Biochem. J. 298:249-258.

2. Nathan, C. 1997. Inducible nitric oxide synthase: what difference does it make? J. Clin. Invest. 100:2417-2423.

3. Kamijo, R., et al. 1994. Requirement for transcription factor IRF-1 in NO synthase induction in macrophages. Science. 263:1612-1615.

4. Xie, Q.W., Kashiwabara, Y., and Nathan, C. 1994. Role of transcription factor NF-kappa B/Rel in induction of nitric oxide synthase. J. Biol. Chem. 269:4705-4708.

5. Stuehr, D.J., Cho, H.J., Kwon, N.S., Weise, M.F., and Nathan, C.F. 1991. Purification and characterization of the cytokine-induced macrophage nitric oxide synthase: an FAD- and FMN-containing flavoprotein. Proc. Natl. Acad. Sci. USA. 88:7773-7777.

6. Vasquez-Vivar, J., et al. 1998. Superoxide generation by endothelial nitric oxide synthase: the influence of cofactors. Proc. Natl. Acad. Sci. USA. 95:9220-9225

7. Xia, Y., and Zweier, J.L. 1997. Superoxide and peroxynitrite generation from inducible nitric oxide synthase in macrophages. Proc. Natl. Acad. Sci. USA. 94:6954-6958.

8. Kojda, G., and Harrison, D. 1999. Interactions between NO and reactive oxygen species: pathophysiological importance in atherosclerosis, hypertension, diabetes and heart failure. Cardiovasc. Res. 43:562-571.

9. Cotton, J.M., et al. 2001. Effects of nitric oxide synthase inhibition on basal function and the force-frequency relationship in the normal and failing human heart in vivo. Circulation. 104:2318-2323.

10. Shimabukuro, M., Ohneda, M., Lee, Y., and Unger, R.H. 1997. Role of nitric oxide in obesity-induced beta cell disease. J. Clin. Invest. 100:290-295.

11. Guslandi, M. 1998. Nitric oxide and inflammatory bowel diseases. Eur. J. Clin. Invest. 28:904-907.

12. Wong, M.L., et al. 1996. Inducible nitric oxide synthase gene expression in the brain during systemic inflammation. Nat. Med. 2:581-584.
13. Balligand, J.L., et al. 1994. Cytokine-inducible nitric oxide synthase (iNOS) expression in cardiac myocytes. Characterization and regulation of iNOS expression and detection of iNOS activity in single cardiac myocytes in vitro. J. Biol. Chem. 269:27580-27588.

14. de Belder, A.J., et al. 1993. Nitric oxide synthase activities in human myocardium. Lancet. 341:84-85.

15. Haywood, G.A., et al. 1996. Expression of inducible nitric oxide synthase in human heart failure. Circulation. 93:1087-1094.

16. Lewis, N.P., et al. 1996. Induction of nitric oxide synthase in the human cardiac allograft is associated with contractile dysfunction of the left ventricle. Circulation. 93:720-729.

17. MacMicking, J.D., et al. 1995. Altered responses to bacterial infection and endotoxic shock in mice lacking inducible nitric oxide synthase [erratum 1995, 81:1170]. Cell. 81:641-650.

18. Koglin, J., Glysing-Jensen, T., Mudgett, J.S., and Russell, M.E. 1998. NOS2 mediates opposing effects in models of acute and chronic cardiac rejection: insights from NOS2-knockout mice. Am. J. Pathol. 153:1371-1376

19. Koglin, J., et al. 1999. Attenuated acute cardiac rejection in NOS2 -/recipients correlates with reduced apoptosis. Circulation. 99:836-842.

20. Szabo, C., Southan, G.J., and Thiemermann, C. 1994. Beneficial effects and improved survival in rodent models of septic shock with Smethylisothiourea sulfate, a potent and selective inhibitor of inducible nitric oxide synthase. Proc. Natl. Acad. Sci. USA. 91:12472-12476.

21. Wildhirt, S.M., et al. 1996. S-methylisothiourea inhibits inducible nitric oxide synthase and improves left ventricular performance after acute myocardial infarction. Biochem. Biophys. Res. Commun. 227:328-333.

22. Nava, E., Noll, G., and Luscher, T.F. 1995. Nitric oxide in cardiovascular diseases. Ann. Med. 27:343-351.

23. Yu, Z., Redfern, C.S., and Fishman, G.I. 1996. Conditional transgene expression in the heart. Circ. Res. 79:691-697.

24. Gossen, M., and Bujard, H. 1992. Tight control of gene expression in mammalian cells by tetracycline-responsive promoters. Proc. Natl. Acad. Sci. USA. 89:5547-5551.

25. Ju, H., et al. 2001. Conditional and targeted over-expression of vascular chymase causes hypertension in transgenic mice. Proc. Natl. Acad. Sci. USA. 98:7469-7474.

26. Weiler-Guettler, H., Aird, W.C., Husain, M., Rayburn, H., and Rosenberg, R.D. 1996. Targeting of transgene expression to the vascular endothelium of mice by homologous recombination at the thrombomodulin locus. Circ. Res. 78:180-187.

27. El-Badawi, A., and Schenk, E.A. 1967. Histochemical methods for separate, consecutive and simultaneous demonstration of acetylcholinesterase and norepinephrine in cryostat sections. J. Histochem. Cytochem. 15:580-588.

28. Dong, R., Liu, P., Wee, L., Butany, J., and Sole, M.J. 1992. Verapamil ameliorates the clinical and pathological course of murine myocarditis. J. Clin. Invest. 90:2022-2030.

29. Nguyen-Tran, V.T., et al. 2000. A novel genetic pathway for sudden cardiac death via defects in the transition between ventricular and conduction system cell lineages. Cell. 102:671-682.

30. Schulz, R., Nava, E., and Moncada, S. 1992. Induction and potential biological relevance of a $\mathrm{Ca}(2+)$-independent nitric oxide synthase in the myocardium. Br. J. Pharmacol. 105:575-580.

31. Jordan, J.E., Zhao, Z.Q., and Vinten-Johansen, J. 1999. The role of neutrophils in myocardial ischemia-reperfusion injury. Cardiovasc. Res. 43:860-878.

32. Svendsen, J.H. 1991. Myocardial capillary permeability for small hydrophilic indicators during normal physiological conditions and after ischemia and reperfusion. Acta. Physiol. Scand. Suppl. 603:119-123.

33. Bia, B.L., et al. 1999. Decreased myocardial nNOS, increased iNOS and abnormal ECGs in mouse models of Duchenne muscular dystrophy. J. Mol. Cell. Cardiol. 31:1857-1862.

34. Brenman, J.E., Chao, D.S., Xia, H., Aldape, K., and Bredt, D.S. 1995. Nitric oxide synthase complexed with dystrophin and absent from skeletal muscle sarcolemma in Duchenne muscular dystrophy. Cell. 82:743-752.

35. Kusama, Y., Bernier, M., and Hearse, D.J. 1990. Exacerbation of reperfusion arrhythmias by sudden oxidant stress. Circ. Res. 67:481-489.

36. Nejima, J., et al. 1989. Superoxide dismutase reduces reperfusion arrhythmias but fails to salvage regional function or myocardium at risk in conscious dogs. Circulation. 79:143-153.

37. Hagendorff, A., Schumacher, B., Kirchhoff, S., Luderitz, B., and Willecke, K. 1999. Conduction disturbances and increased atrial vulnerability in Connexin 40-deficient mice analyzed by transesophageal stimulation. Circulation. 99:1508-1515.

38. Nakamura, K., et al. 2001. Complete heart block and sudden death in mice overexpressing calreticulin. J. Clin. Invest. 107:124-153.

39. Heger, J., et al. 2002. Cardiac-specific overexpression of inducible nitric oxide synthase does not result in severe cardiac dysfunction. Circ. Res. 90:93-99. 